\title{
DIFFERENTIABILITY OF PEANO DERIVATIVES
}

\author{
ANDREAS FISCHER
}

(Communicated by David Preiss)

\begin{abstract}
Peano differentiability is a notion of higher-order Fréchet differentiability. H. W. Oliver gave sufficient conditions for the $m^{\text {th }}$ Peano derivative to be a Fréchet derivative in the case of functions of a real variable. Here we generalize this theorem to functions of several variables.
\end{abstract}

\section{INTRODUCTION}

In 1891, G. Peano introduced the class of functions which can be best approximated by a polynomial of degree less than or equal to $m$. These functions are called Peano differentiable. For a precise definition we use the following conventions. For a multi-index $\alpha \in \mathbb{N}^{n}$ we set $|\alpha|=\alpha_{1}+\cdots+\alpha_{n}, \alpha !=\alpha_{1} ! \cdots \alpha_{n}$ !, and, if $x \in \mathbb{R}^{n}$, $x^{\alpha}=x_{1}^{\alpha_{1}} \cdots x_{n}^{\alpha_{n}}$. The notion of Peano differentiability reads as follows.

Definition 1.1. Let $U \subset \mathbb{R}^{n}$ be an open set and $m$ a positive integer. The function $f: U \rightarrow \mathbb{R}$ is called $m$ times Peano differentiable at $x_{0} \in U$ if there are $f_{\alpha}\left(x_{0}\right) \in \mathbb{R}$, $|\alpha| \leq m$, such that

$$
\lim _{x \rightarrow x_{0}} \frac{f(x)-\sum_{|\alpha| \leq m} \frac{f_{\alpha}\left(x_{0}\right)}{\alpha !}\left(x-x_{0}\right)^{\alpha}}{\left\|x-x_{0}\right\|^{m}}=0 .
$$

A function with codomain $\mathbb{R}^{p}$ is called $m$ times Peano differentiable if each coordinate function possesses this property. The $f_{\alpha}\left(x_{0}\right)$ are called Peano derivatives of order $|m|$. The set of all $m$ times Peano differentiable functions with domain $U$ forms a ring, and, moreover, the composition of $m$ times Peano differentiable functions is again $m$ times Peano differentiable.

The case $m=1$ obviously represents the notion of Fréchet differentiability so that we can interpret the notion for $m>1$ as differentiability of higher order.

Example 1.2. Let $m>1$ be an integer and the function $f: \mathbb{R} \rightarrow \mathbb{R}$ be defined by $f(x):=x^{m+1} \sin \left(x^{-m}\right)$ for $x \neq 0$ and $f(0):=0 . f$ is $m$ times Peano differentiable in $\mathbb{R}$, but the first derivative is not continuous.

The proof is straightforward and we omit it.

As a consequence we get that, in general, the $m^{\text {th }}$ Peano derivatives are not derivatives in the usual sense. Conversely, Taylor's Theorem states that $\mathcal{C}^{m}$ functions are $m$ times Peano differentiable.

Received by the editors October 28, 2005 and, in revised form, April 17, 2006.

2000 Mathematics Subject Classification. Primary 26 B05.

The author's research was supported by EC-IHP-Network RAAG (Contract-No: HPRN-CT2001-00271). 
H. W. Oliver found sufficient conditions which guarantee Fréchet differentiability. In [3] he proved the following statement.

Theorem 1.3 (Oliver). Let $I$ be an interval in $\mathbb{R}$ and let $f: I \rightarrow \mathbb{R}$ be an $m$ times Peano differentiable function. If the $m^{\text {th }}$ Peano derivative is locally bounded from above or below at $x_{0} \in I$, the Peano derivative of order $m-1$ is Fréchet differentiable at $x_{0}$.

We will use Oliver's theorem for the study of $m^{\text {th }}$ directional derivatives of $m$ times Peano differentiable functions, and we will prove that they are Fréchet derivatives if they are bounded from above or below. Knowing this fact we can deduce the following theorem.

Theorem 1.4. Let $U \subset \mathbb{R}^{n}$ be an open subset and let $f: U \rightarrow \mathbb{R}$ be an $m$ times Peano differentiable function. Let the $m^{\text {th }}$ Peano derivatives all be bounded from above or all be bounded from below. Then the Peano derivatives of order $m-1$ are Fréchet differentiable at each $x_{0} \in U$.

In [1] it is shown that if $f$ is $m$-convex and $m$ times Peano differentiable, it is $m$ times Fréchet differentiable. For Peano differentiable functions of several variables and $m \geq 3, m$-convexity is neither more general nor more special than the conditions given in Theorem 1.4. In the case $m=2$ we need weaker conditions for obtaining Fréchet differentiability of the first derivatives; cf. Corollary 4.1, which is already known (personal communication with Clifford Weil).

\section{Preliminary LEMmas}

The first lemma of this section deals with the number of zeros of the derivatives of a $p$ times differentiable map $f$ of one real variable. We compute an upper bound for the number of zeros of the derivatives under the assumption that the derivative of order $p$ is positive.

Lemma 2.1. Let $h:(a, b) \rightarrow \mathbb{R}$ be $p$ times differentiable and

$$
h^{(p)}(t)>0, \quad t \in(a, b) .
$$

Then there is a subinterval $(c, d) \subset(a, b)$ of length

$$
|d-c| \geq|b-a| / p^{2}
$$

such that all derivatives of order $2, \ldots, p$ are either positive or negative on $(c, d)$.

Proof. We show by induction on $j$ that for $j=0, \ldots, p-1$ the number of zero-points of $h^{(p-j)}$ is bounded by $j$ :

$j=0$ :

$h^{(p-0)}$ is a priori positive. Therefore it has no zero-point.

$j \rightsquigarrow j+1$ :

By the assumption, $h^{(p-j)}$ has at most $j$ zero-points $x_{1}<x_{2}<\cdots<x_{j}$ in $(a, b)$.

We put $x_{0}=a$ and $x_{j+1}=b$ so that on each of the $j+1$ intervals $\left(x_{l}, x_{l+1}\right)$ where $l=0, \ldots, j$, the $(p-j)^{\text {th }}$ derivative of $h$ is strictly positive or negative.

Hence, $\left.h^{(p-(j+1))}\right|_{\left(x_{l}, x_{l+1}\right]}$ is a strictly monotone function and may therefore have at most one zero-point.

So there are at most $\sum_{l=0}^{p-1} l=\frac{p(p-1)}{2} \leq p^{2}$ points at which at least one of the $h^{(i)}$ equals 0 where $i=2, \ldots, p$. Hence, we find an interval $(c, d) \subset(a, b)$ of length

$$
|b-a| / p^{2}
$$


such that for $i=2, \ldots, p$, the $i^{\text {th }}$ derivative of $h$ is either positive or negative.

Now we focus on $p$ times differentiable functions of one variable with definite derivatives. We find the next two lemmas in [2]. Here we give alternative proofs.

Lemma 2.2. Let $I \subset \mathbb{R}$ be an interval, $\lambda: I \rightarrow \mathbb{R}$ be twice differentiable, and we have either $\lambda^{(2)} \geq 0$ on $I$ or $\lambda^{(2)} \leq 0$ on I. If $t \in I$ and $r>0$ such that $[t-r, t+r] \subset I$, then

$$
\left|\lambda^{(1)}(t)\right| \leq \frac{2}{r} \sup _{s \in(t-r, t+r)}|\lambda(s)| .
$$

Proof. Without loss of generality we may assume that $\lambda^{(2)} \leq 0$ on $I$ so that $\lambda^{(1)}$ is monotonically decreasing on $I$. According to the Mean-Value Theorem we obtain the following two inequalities:

$$
\begin{aligned}
& \lambda(t)-\lambda(t-r) \geq \inf _{s \in[t-r, t]} \lambda^{(1)}(s) r=\lambda^{(1)}(t) r, \\
& \lambda(t+r)-\lambda(t) \leq \sup _{s \in[t, t+r]} \lambda^{(1)}(s) r=\lambda^{(1)}(t) r .
\end{aligned}
$$

Since $|\lambda(t)-\lambda(t-r)|$ and $|\lambda(t+r)-\lambda(t)|$ are both less than or equal to

$$
2 \sup _{s \in[t-r, t+r]}|\lambda(s)|
$$

we get

$$
\left|\lambda^{(1)}(t)\right| \leq \frac{2}{r} \sup _{s \in(t-r, t+r)}|\lambda(s)| .
$$

This lemma generalizes to the next one.

Lemma 2.3. Let $\lambda: I \rightarrow \mathbb{R}$ be $p$ times differentiable such that for $i=2, \ldots, p$, we have either $\lambda^{(i)} \geq 0$ on $I$ or $\lambda^{(i)} \leq 0$ on $I$. Let $t \in I$ and $r>0$ such that $[t-r, t+r] \subset I$. Then, for $j=1, \ldots, p-1$, there is a $C_{j}>0$ such that

$$
\left|\lambda^{(j)}(t)\right| \leq r^{-j} C_{j} \sup _{s \in[t-r, t+r]}|\lambda(s)| .
$$

Proof. We proceed by induction on $j$. The case $j=1$ was proved in Lemma 2.2 with $C_{1}=2$. So we assume that the statement of the lemma holds true for $j$. Let $u \in\left[t-\frac{r}{2}, t+\frac{r}{2}\right] \subset[t-r, t+r] \subset I$. Then the interval $\left[u-\frac{r}{2}, u+\frac{r}{2}\right] \subset I$, and we obtain

$$
\left|\lambda^{(j)}(u)\right| \leq C_{j}\left(\frac{r}{2}\right)^{-j} \sup _{s \in[t-r, t+r]}|\lambda(s)| .
$$

Since $\lambda^{(j+2)}:\left[t-\frac{r}{2}, t+\frac{r}{2}\right] \rightarrow \mathbb{R}$ is non-negative or non-positive we can apply Lemma 2.2 to $\lambda^{(j)}$ and get with $C_{j+1}:=C_{j} 2^{j+2}$,

$$
\begin{aligned}
\left|\lambda^{(j+1)}(t)\right| & \leq \frac{2}{\left(\frac{r}{2}\right)} \sup _{u \in\left[t-\frac{r}{2}, t+\frac{r}{2}\right]}\left|\lambda^{(j)}(u)\right| \\
& \leq \frac{4}{r} C_{j}\left(\frac{r}{2}\right)^{-j} \sup _{s \in[t-r, t+r]}|\lambda(s)| \\
& =C_{j+1} r^{-(j+1)} \sup _{s \in[t-r, t+r]}|\lambda(s)| .
\end{aligned}
$$

As a consequence of Lemma 2.1 and Lemma 2.3 we obtain 
Corollary 2.4. Let $h:(a, b) \rightarrow \mathbb{R}$ be $p$ times differentiable and

$$
h^{(p)}(t)>0, \quad t \in(a, b) .
$$

Then, there is a constant $\tilde{C}_{j}$ depending only on $j$, and a subinterval $(c, d) \subset(a, b)$ of length

$$
|d-c| \geq \frac{|b-a|}{p^{2}}
$$

such that for $j=1, \ldots, p-1$ :

$$
\left|h^{(j)}(s)\right| \leq \tilde{C}_{j} \sup _{t \in(c, d)} \frac{|h(t)|}{|d-c|^{j}}, \quad s \in\left(c+\frac{d-c}{4}, d-\frac{d-c}{4}\right) .
$$

Proof. According to Lemma 2.1 we get a subinterval $(c, d) \subset(a, b)$ of the desired length where each of the derivatives of $h$ is definite. We apply Lemma 2.3 to $h$ restricted to $(c, d)$ with $r=(d-c) / 4$ and $t \in\left(c+\frac{d-c}{4}, d-\frac{d-c}{4}\right)$.

\section{Directional Derivatives}

In this section we consider $(m-1)^{\text {th }}$ directional derivatives of $m$ times Peano differentiable functions where $m \geq 2$. Here we interpret higher-order directional derivatives in the sense of Peano.

Lemma 3.1. Let $U \subset \mathbb{R}^{n}$ be open, let $f: U \rightarrow \mathbb{R}$ be an $m$ times Peano differentiable function and $\nu \in \mathbb{R}^{n}$ a unit vector. If the $m^{\text {th }}$ directional derivative of $f$ with respect to $\nu$ is locally bounded from below (above) at $x_{0} \in U$, the corresponding $(m-1)^{\text {th }}$ directional derivative is Fréchet differentiable at $x_{0}$.

Proof. We denote by $B(r)$ the ball with centre 0 and radius $r$. Without loss of generality we may assume that $U=B(1), x_{0}=0$, and all Peano derivatives up to order $m$ vanish at 0 . Furthermore, we may assume that the $m^{\text {th }}$ directional derivative with respect to $\nu$ is bounded from below on $B(1)$ by $K<0$.

We show that the mapping

$$
x \mapsto \frac{\partial^{m-1}}{\partial \nu^{m-1}} f(x)
$$

is $o(\|x\|)$.

In order to show this we assume that the mapping above is not $o(\|x\|)$; e.g., there is an $L>0$ and a zero-sequence $\left(y_{l}\right)_{l \in \mathbb{N}}$ such that

$$
\forall l \frac{\partial^{m-1}}{\partial \nu^{m-1}} f\left(y_{l}\right) \geq L\left\|y_{l}\right\| \quad\left(\text { or } \leq-L\left\|y_{l}\right\|\right) .
$$

Step 1 . We consider the following family of functions $g_{l}:\left[-\frac{1}{2}, \frac{1}{2}\right] \rightarrow \mathbb{R}, l \in \mathbb{N}$ with

$$
g_{l}(t):=f\left(y_{l}+t \nu\right) .
$$

Since $g_{l}$ is an $m$ times Peano differentiable function of the variable $t$ such that the derivative of order $m$ is bounded from below, we know by Oliver's theorem that $g_{l}$ is $m$ times Fréchet differentiable in $\left[-\frac{1}{2}, \frac{1}{2}\right]$.

We may apply the Mean-Value Theorem to the $(m-1)^{\text {th }}$ derivative of $g_{l}$. If $s \geq 0$,

$$
g_{l}^{(m-1)}(s)-g_{l}^{(m-1)}(0) \geq K(s-0)
$$


since $K$ is a lower bound for the $m^{\text {th }}$ derivative of $g_{l}$, which is in fact the function $t \mapsto \frac{\partial^{m} f}{\partial \nu^{m}}\left(y_{l}+t \nu\right)$.

Hence, if $0 \leq s \leq \frac{L\left\|y_{l}\right\|}{-2 K}$,

$$
\begin{aligned}
g_{l}^{(m-1)}(s) & =g_{l}^{m-1}(0)+K s \\
& \geq g_{l}^{(m-1)}(0)-\frac{L\left\|y_{l}\right\|}{2} \\
& =\frac{\partial^{m-1}}{\partial \nu^{m-1}} f\left(y_{l}\right)-\frac{L\left\|y_{l}\right\|}{2} \\
& \geq \frac{L\left\|y_{l}\right\|}{2} .
\end{aligned}
$$

In particular, $g_{l}^{(m-1)}$ is positive on $\left(0, \frac{L\left\|y_{l}\right\|}{-2 K}\right)$.

Step 2. We apply Corollary 2.4 to $g_{l}$ restricted to the interval $\left(0, \frac{L\left\|y_{l}\right\|}{-2 K}\right)$ with $p=m-1$.

Thus, there is a subinterval $\left(c_{l}, d_{l}\right) \subset\left(0, \frac{L\left\|y_{l}\right\|}{-2 K}\right)$ such that

$$
\left|g_{l}^{(m-2)}(s)\right| \leq \tilde{C}_{m-2} \sup _{s^{\prime} \in\left(c_{l}, d_{l}\right)} \frac{\left|g_{l}\left(s^{\prime}\right)\right|}{\left|d_{l}-c_{l}\right|^{m-2}}
$$

whenever $s \in\left(c_{l}+\frac{d_{l}-c_{l}}{4}, d_{l}-\frac{d_{l}-c_{l}}{4}\right)$. Moreover, we may assume that for $c_{l}$ and $d_{l}$,

$$
\left|d_{l}-c_{l}\right|=\frac{L\left\|y_{l}\right\|}{-2 K(m-1)^{2}} .
$$

So, for $s \in\left(c_{l}+\frac{d_{l}-c_{l}}{4}, d_{l}-\frac{d_{l}-c_{l}}{4}\right)$ we obtain the inequality

$$
\begin{aligned}
\left|g_{l}^{(m-2)}(s)\right| & \leq N_{1} \sup _{s^{\prime} \in\left(c_{l}, d_{l}\right)} \frac{\left|g_{l}\left(s^{\prime}\right)\right|}{\left\|2 y_{l}\right\|^{m-2}} \\
& \leq N \sup _{z \in B\left(2\left\|y_{l}\right\|\right)} \frac{f(z)}{\|z\|^{m-2}}
\end{aligned}
$$

where $N_{1}$ and $N$ are positive constants depending only on $m, L, K$.

Step 3a. Since $g_{l}^{(m-2)}$ is continuous on the closed interval $\left[\frac{-1}{2}, \frac{1}{2}\right]$ we conclude that for $s_{l}:=c_{l}+\frac{d_{l}-c_{l}}{4}$ and $t_{l}:=d_{l}-\frac{d_{l}-c_{l}}{4}$,

$$
\left|g_{l}^{(m-2)}\left(t_{l}\right)-g_{l}^{(m-2)}\left(s_{l}\right)\right| \leq 2 N \sup _{z \in B\left(2\left\|y_{l}\right\|\right)} \frac{f(z)}{\|z\|^{m-2}} .
$$

Step 3b. According to the Mean-Value Theorem in connection with equation (3.5) we can give an alternative estimate for $\left|g_{l}^{(m-2)}\left(t_{l}\right)-g_{l}^{(m-2)}\left(s_{l}\right)\right|$ :

$$
\begin{aligned}
\left|g_{l}^{(m-2)}\left(t_{l}\right)-g_{l}^{(m-2)}\left(s_{l}\right)\right| & \geq\left(t_{l}-s_{l}\right) \inf _{u \in\left(s_{l}, t_{l}\right)} g_{l}^{(m-1)}(u) \\
& \geq\left(t_{l}-s_{l}\right) \frac{L\left\|y_{l}\right\|}{2} \\
& =\frac{L\left\|y_{l}\right\|}{-2 K(m-1)^{2}} \frac{L\left\|y_{l}\right\|}{2} \\
& \geq \tilde{N}\left\|y_{l}\right\|^{2}
\end{aligned}
$$

with a positive constant $\tilde{N}$ depending only on $m, L, K$. 
Step 4. Since $f$ is $o\left(\|x\|^{m}\right)$ there is for each $l_{0} \in \mathbb{N}$, an $l \geq l_{0}$ such that

$$
\sup _{z \in B\left(2\left\|y_{l}\right\|\right)} \frac{f(z)}{\|z\|^{m-2}} \leq \frac{\tilde{N}}{4 N}\left\|y_{l}\right\|^{2} .
$$

Steps $3 \mathrm{a}$ and $3 \mathrm{~b}$ now imply for this $l$ that

$$
\frac{\tilde{N}}{2}\left\|y_{l}\right\|^{2} \geq 2 N \sup _{z \in B\left(2\left\|y_{l}\right\|\right)} \frac{f(z)}{\|z\|^{m-2}} \geq\left|g_{l}^{(m-2)}\left(t_{l}\right)-g_{l}^{(m-2)}\left(s_{l}\right)\right| \geq \tilde{N}\left\|y_{l}\right\|^{2},
$$

which is a contradiction.

So, the assumption of (3.2) is false.

Step 5. We can show analogously that the assumption

$$
\forall l \frac{\partial^{m-1}}{\partial \nu^{m-1}} f\left(y_{l}\right) \leq-L\left\|y_{l}\right\|
$$

leads to a contradiction.

Hence, $\frac{\partial^{m-1}}{\partial \nu^{m-1}} f(x)$ is $o(\|x\|)$; i.e., it is Fréchet differentiable at 0 .

We note that in the case of $\nu$ being one of the standard unit vectors $e_{i} \in \mathbb{R}^{n}$, the directional derivatives of $f$ with respect to $\nu$ are the corresponding partial derivatives.

\section{Results}

Lemma 3.1 leads us directly to the following corollary.

Corollary 4.1. Let $U \subset \mathbb{R}^{n}$ be open and $f: U \rightarrow \mathbb{R}$ a 2 times Peano differentiable function. If every second partial derivative of $f$ is locally bounded from above or below at $x_{0} \in U$, then $f$ is 2 times Fréchet differentiable at $x_{0}$.

This corollary cannot directly be generalized to higher-order Peano differentiable functions. This is due to the fact that for $m \geq 3$ the Peano derivatives of order $m-1$ can be mixed derivatives; i.e., they are no longer directional derivatives. But, by some stronger assumptions, as they are given in Theorem 1.4, we can obtain an analogous statement for $m \geq 3$.

Proof of Theorem 1.4. If $\nu \in\left(\mathbb{R}_{0}^{+}\right)^{n}, t \in \mathbb{R}$, then

$$
f(x+t \nu)=\sum_{|\alpha| \leq m} \frac{f_{\alpha}(x)}{\alpha !} \nu^{\alpha} t^{|\alpha|}+o\left(t^{m}\right)
$$

cf. equation (1.1). So the $k^{\text {th }}$ directional derivative with respect to $\nu$ at the point $x$ is given by

$$
\sum_{|\alpha|=k} \frac{k !}{\alpha !} f_{\alpha}(x) \nu^{\alpha}
$$

Since for each $\alpha$ with $|\alpha|=m, \frac{m !}{\alpha !} \nu^{\alpha}$ is non-negative and the $f_{\alpha}$ are bounded from below (above), the $m^{\text {th }}$ directional derivative of $f$ with respect to $\nu$ is bounded from below (above) so that Lemma 3.1 implies Fréchet differentiability for $\frac{\partial^{m-1}}{\partial \nu^{m-1}} f(x)$.

We show that every $(m-1)^{\text {th }}$ Peano derivative of $f$ is a linear combination of the $(m-1)^{\text {th }}$ directional derivatives with direction $\nu \in\left(\mathbb{R}_{0}^{+}\right)^{n}$. Let $\beta(1), \ldots, \beta(s)$ denote the multi-indices $\alpha \in \mathbb{N}^{n}$ with $|\alpha|=m-1$. It remains to show the existence of $s$ 
linearly independent vectors $w_{l}=\left(\nu_{l}^{\beta(1)}, \ldots, \nu_{l}^{\beta(s)}\right)$ with unit vectors $\nu_{l} \in\left(\mathbb{R}_{0}^{+}\right)^{n}$, $l=1, \ldots, s$. We select a sequence $p_{1}<\cdots<p_{n}$ of prime numbers, and we put $p(l):=\left(p_{1}^{l}, \ldots, p_{n}^{l}\right)$. Then we set $\nu_{l}:=p(l) /\|p(l)\|$, so $\nu_{l} \in\left(\mathbb{R}_{0}^{+}\right)$is a unit vector.

Claim: The vectors $w_{l}:=\left(\nu_{l}^{\beta(1)}, \ldots, \nu_{l}^{\beta(s)}\right), l=1, \ldots, s$, are linearly independent.

We consider the vectors $v_{l}:=\|p(l)\|^{m-1} w_{l}=\left(\left(p^{\beta(1)}\right)^{l}, \ldots,\left(p^{\beta(s)}\right)^{l}\right)$, so

$$
V:=\left(\begin{array}{c}
v_{1} \\
\vdots \\
v_{s}
\end{array}\right)
$$

is a matrix of Vandermonde type. The uniqueness of prime decomposition implies for $i \neq j, p^{\beta(i)} \neq p^{\beta(j)}$, so $V$ is invertible. Therefore, the $v_{l}$ are linearly independent, so the $w_{l}$ are linearly independent, so the claim is proved.

Hence, each $f_{\alpha},|\alpha|=m-1$, is a linear combination of Fréchet differentiable functions, so they are Fréchet differentiable, too.

We note a consequence of Theorem 1.4.

Corollary 4.2. Let $U \subset \mathbb{R}^{n}$ be an open subset and $f: U \rightarrow \mathbb{R}$ be an $m$ times Peano differentiable function. Let the $m^{\text {th }}$ Peano derivatives all be locally bounded at $x_{0} \in U$. Then the $(m-1)^{\text {th }}$ Peano derivatives are Fréchet differentiable at $x_{0}$.

\section{REFERENCES}

1. Dayal, Saheb, Higher Fréchet and discrete Gâteaux differentiability of $n$-convex functions on Banach spaces. Functional analysis and operator theory (New Delhi, 1990), 154-171, Lecture Notes in Math., 1511, Springer, Berlin, 1992. MR1180760 (93h:58014)

2. Kurdyka, Krzysztof; Pawłucki, Wiesław, Subanalytic version of Whitney's extension theorem. Studia Math. 124 (1997), no. 3, 269-280. MR1456425 (98i:32008)

3. Oliver, H. William, The exact Peano derivative. Trans. Amer. Math. Soc. 76 (1954), 444-456. MR0062207 (15:944d)

4. Peano, G., Sulla formula di Taylor. (Italian) Torino Atti XXVII. 40-46. (1891).

Department of Mathematics, University of Saskatchewan, 106 Wiggins Road, SaskaTOOn, Saskatchewan S7N 5E6, Canada

E-mail address: el.fischerandreas@web.de 